\title{
Gender determination: Role of lip prints, finger prints and mandibular canine index
}

\author{
RESHMA POOTHAKULATH KRISHNAN ${ }^{1 *}$, RADHIKA THANGAVELU $^{2 *}$, \\ VIDHYA RATHNAVELU ${ }^{1}$ and MALATHI NARASIMHAN ${ }^{1}$ \\ ${ }^{1}$ Department of Oral and Maxillofacial Pathology, Faculty of Dental Science, Sri Ramachandra University, Chennai 600116; \\ ${ }^{2}$ Department of Oral Pathology, Thai Moogambigai Dental College and Hospital, Mugappair, Chennai 600107, India
}

Received January 22, 2015; Accepted November 30, 2015

DOI: $10.3892 /$ etm.2016.3245

\begin{abstract}
Personal identification has a pivotal role in forensic investigations. Gender determination is an essential step in personal identification. Despite the advent of advanced techniques such as DNA fingerprinting, methods such as lip print and fingerprint analysis and mandibular canine index calculations are routinely used in gender determination, as they are simple and cost-effective. The present study investigated the hypothesis that lip print analysis is an effective tool in gender determination compared with fingerprint analysis and the mandibular canine index. The predominant patterns of lip prints and fingerprints were analyzed in males and females, and the efficacy of the mandibular canine index in gender determination was evaluated. The study group comprised 50 students, 25 males and 25 females who were 18-25 years of age. Lip prints and fingerprints were obtained and classified according to Tsuchihashi's classification and Kücken and Newell's classification, respectively. Mandibular impressions were made and the mandibular canine index was calculated. Type I and Type I' lip prints were predominant in females, and Type IV lip prints were predominant in males. The analysis of fingerprints revealed that the loop fingerprint pattern was predominant in both males and females. The mandibular canine index was not found to be significant in gender identification. The predominant patterns of lip prints were distinct for males and females; conversely, fingerprints were demonstrated to be similar in both genders. Therefore, lip prints hold an increased potential for gender determination, as compared with fingerprints, and the mandibular canine index is not a reliable indicator of gender.
\end{abstract}

Correspondence to: Dr Vidhya Rathnavelu, Department of Oral and Maxillofacial Pathology, Faculty of Dental Science, Sri Ramachandra University, No. 1, Sri Ramachandra Nagar, Poonamallee High Road, Porur, Chennai 600116, India

E-mail: drvidhyamds@gmail.com

*Contributed equally

Key words: lip prints, finger prints, mandibular canine index, gender

\section{Introduction}

Dentistry is important in the detection and resolution of crime and civil proceedings, and personal identification has an important role in forensic sciences. Personal identification methods are used for the identification of unrecognizable deceased individuals in mass disasters, decomposed bodies, victims of transport accidents, house fires, missing persons and in child abuse cases $(1,2)$. Gender identification is an important component of personal identification.

Lip prints are defined as the normal lines and fissures present in the form of wrinkles and grooves that are located in the transition zone of the human lip, between the inner labial mucosa and the outer skin (3), the examination of which is referred to as cheiloscopy (4). Lip prints were first reported by Fischer et al in 1902 (5). Lip prints are unique to each person and lip groove patterns rarely change, resisting numerous types of affliction, including physical injury, pressure and exposure to hot or cold stimuli (6). Its uniqueness $(6,7)$ and permanence (6) makes the lip print a reliable tool in gender determination. The term fingerprint refers to the impression of epidermal ridges in the fleshy distal portion of a finger. A fingerprint helps in establishing identification, and is obtained by pressing the finger on paper following the application of ink (8). Dermatoglyphics refers to the study of fingerprints (9). Fingerprint patterns are genotypically determined and remain unchanged throughout life (10). Teeth are durable and resist high temperatures, and are therefore important for forensic investigations. Mandibular canines show the greatest sexual dimorphism (11). The durability and gender dichotomy of the mandibular canines result in them being the key teeth in personal identification $(11,12)$. Canines are the least frequently extracted teeth due to their relatively low susceptibility to dental caries and periodontal disease (13).

The aim of the present study was to analyze the predominant patterns of lip and fingerprints in males and females and to evaluate the efficacy of the mandibular canine index in gender determination.

\section{Materials and methods}

Study sample. The study sample comprised 50 students from Sri Ramachandra University (Chennai, India) including 25 males 
and 25 females of 18-25 years of age. The study was approved by the institutional ethics committee of Sri Ramachandra University. Individuals with no lip or finger pathology, healthy periodontium and dental cavity-free canines were included in the present study. The experimental protocol for obtaining the lip prints, fingerprints and mandibular impression was explained to the study subjects, and written-informed consent was obtained from all study subjects. Each experimental sample was assigned a code to avoid observer bias.

Collection of lip prints. The lips of the participants were thoroughly cleaned using wet tissue paper and allowed to dry. The lips were then outlined using a sharp lip liner pencil. Dark red colored lip stick was applied uniformly. An impression of the lips was made on a cellophane tape and transferred to white bond paper. The impression was analyzed using a magnifying lens. The 50 samples were classified as Type I, Type I', Type II, Type III, Type IV or Type V according to Tsuchihashi's classification (14). Tsuchihashi's classification of the lip print samples was performed as follows: Type I, clear-cut grooves running vertically across the lips; Type I', straight grooves which disappear half way instead of covering the entire lip; Type II, fork grooves in their course; Type III, intersecting grooves; Type IV, reticulate grooves; Type V, undetermined.

Collection of fingerprints. Left hand thumb impressions were obtained on a white sheet. Each subject was asked to wash their hands thoroughly with soap and water and dry them using towel prior to pressing their finger on a Golden Stamp Pad (Ashoka Marketing Co., New Delhi, India) and subsequently creating a fingerprint impression on the paper. A magnifying lens was used to visualize the fingerprints. The 50 samples were classified according to the classification of Kücken and Newell (9). This classification of the fingerprint samples was performed according to the characteristics of the prints as follows: Type 1, loop pattern; Type 2, arch pattern; Type 3, whorl pattern.

Determination of mandibular canine index. Mandibular impressions were made using alginate (Alginate-Algitex; Bombay Burmah Trading Corporation, Ltd., Mumbai, India), and study models were prepared using dental stone (Goldstone; Asian Chemicals, Rajkot, India), as previously described (12). The mesio-distal width on either side of the arch and inter canine width of all 50 models were calculated using digital vernier calipers (Bliss Classic, Yamayo, India) (Fig. 1). The observed mandibular canine index $\left(\mathrm{MCI}_{\mathrm{o}}\right)$ was calculated using the following formula:

\section{$\mathrm{MCl}_{0}=$ Average mesio-distal crown width of mandibular canine Inter canine width}

Standard mandibular canine index $\left(\mathrm{MCI}_{\mathrm{s}}\right)$ was calculated from mean mandibular canine index (MCI) and standard deviation (SD) values in males and females using the following formula:

$$
\mathrm{MCl}_{\mathrm{S}}=\frac{(\text { Mean male } \mathrm{MCl}-\mathrm{SD})+(\text { Mean female } \mathrm{MCl}+\mathrm{SD})}{\text { (M) }}
$$
2

The $\mathrm{MCI}_{\mathrm{s}}$ was used as a cut-off point to differentiate between males and females (12).
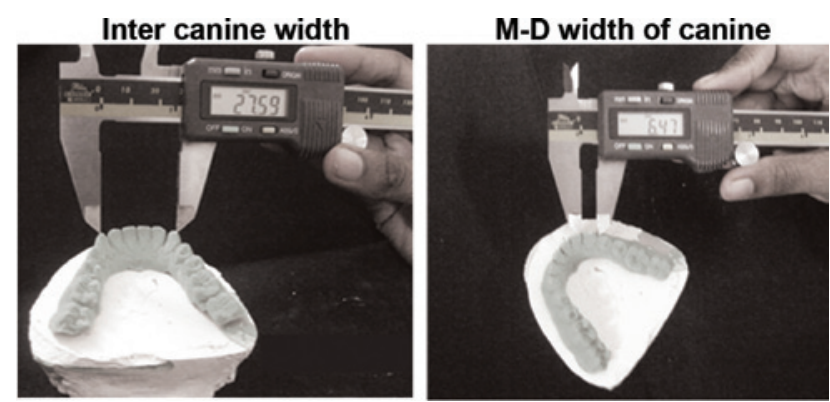

Figure 1. Estimation of mandibular canine index and measurement of the inter-canine width and M-D width of canine. M-D, mesiodistal.

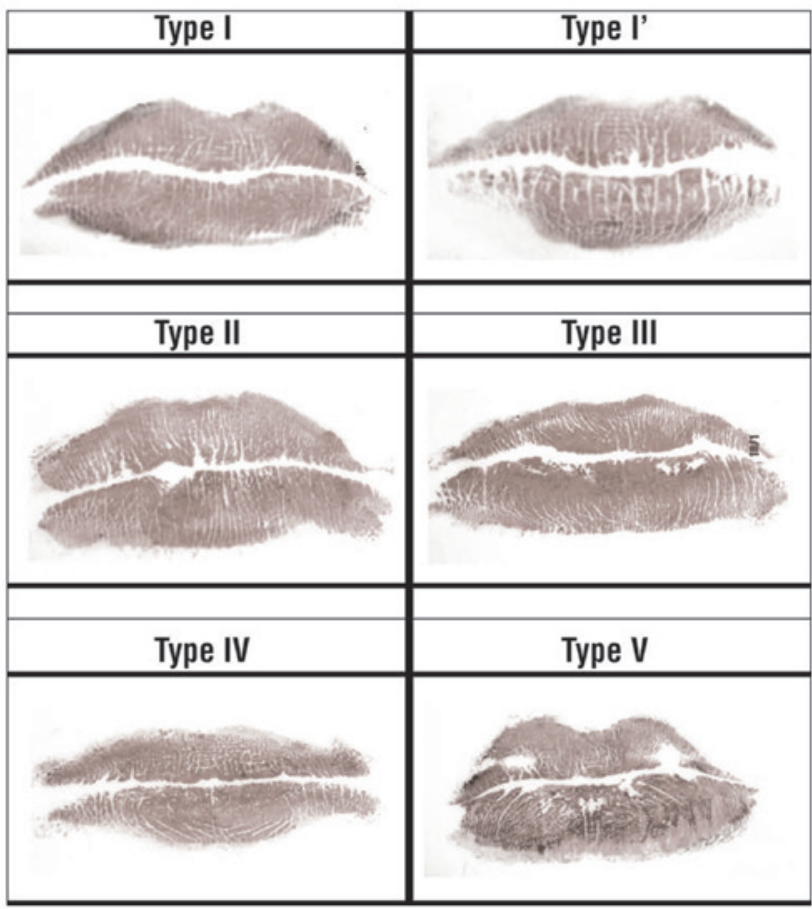

Figure 2. Patterns of lip prints in the present study.

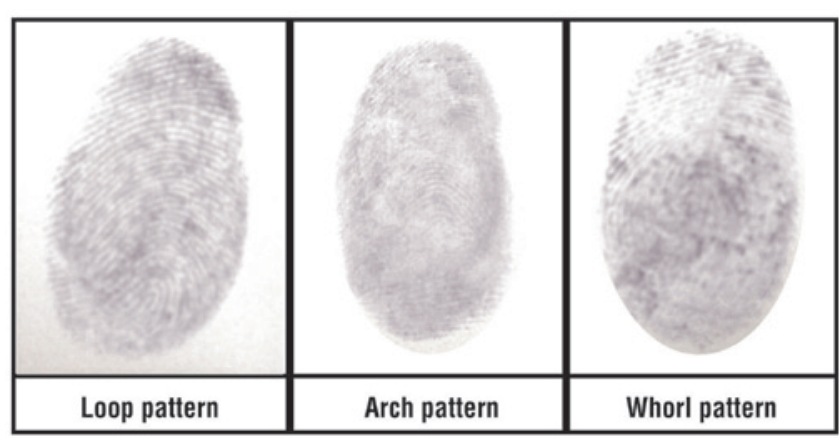

Figure 3. Patterns of fingerprints in the present study.

Statistical analysis. Statistical analysis was performed using a $\chi^{2}$ test. An unpaired t-test was used to analyze differences in parameters between the two genders. Data were analyzed using SPSS software (version 15.0; SPSS, Inc., Chicago, IL, USA). $\mathrm{P} \leq 0.05$ was considered to indicate a statistically significant result. 
Table I. Gender determined by MCI.

\begin{tabular}{cccc}
\hline & \multicolumn{2}{c}{ Biological gender } & \\
\cline { 2 - 3 } Gender as determined by MCI & Male & Female & Total \\
\hline Male MCI gender, $\mathrm{n}$ & 21 & 19 & 40 \\
\% within male MCI gender & 52.5 & 47.5 & 100.0 \\
$\%$ within biological males & 84.0 & 76.0 & 80.0 \\
Female MCI gender, $\mathrm{n}$ & 4 & 6 & 10 \\
\% within female MCI gender & 40.0 & 60.0 & 100.0 \\
$\%$ within biological females & 16.0 & 24.0 & 20.0 \\
Both MCI genders, $\mathrm{n}$ & 25 & 25 & 50 \\
$\%$ within total sample & 50.0 & 50.0 & 100.0 \\
$\%$ within biological gender & 100.0 & 100.0 & 100.0 \\
\hline
\end{tabular}

MCI, mandibular canine index.

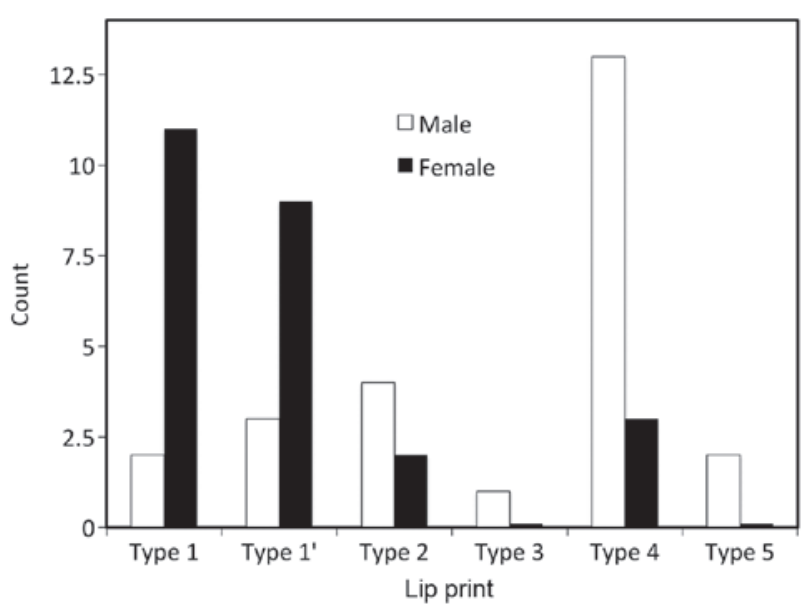

Figure 4. Distribution of lip print patterns in males and females.

\section{Results}

Lip prints and fingerprints. Some examples of patterns of the lip prints and fingerprints of each of the different types investigated in the present study are shown in Figs. 2 and 3, respectively.

Examination of the lip print patterns revealed the following: i) No two lip prints matched each other, establishing the uniqueness of lip prints in personal identification; ii) Type I and Type I' were the predominant pattern types in females (Fig. 4); and iii) Type IV was predominant in males (Fig. 4). The examination of fingerprint patterns revealed the following: i) No two fingerprint patterns matched each other, establishing the uniqueness of fingerprints in personal identification; and ii) the loop pattern of fingerprint was predominant in both males and females (Fig. 5).

Mandibular canine index. The standard mandibular canine index value was calculated in order to investigate the efficacy of the mandibular canine index as a gender determinant; its value was 0.25 . This value was used as the cut-off point, above which the value was considered to indicate a male, and below

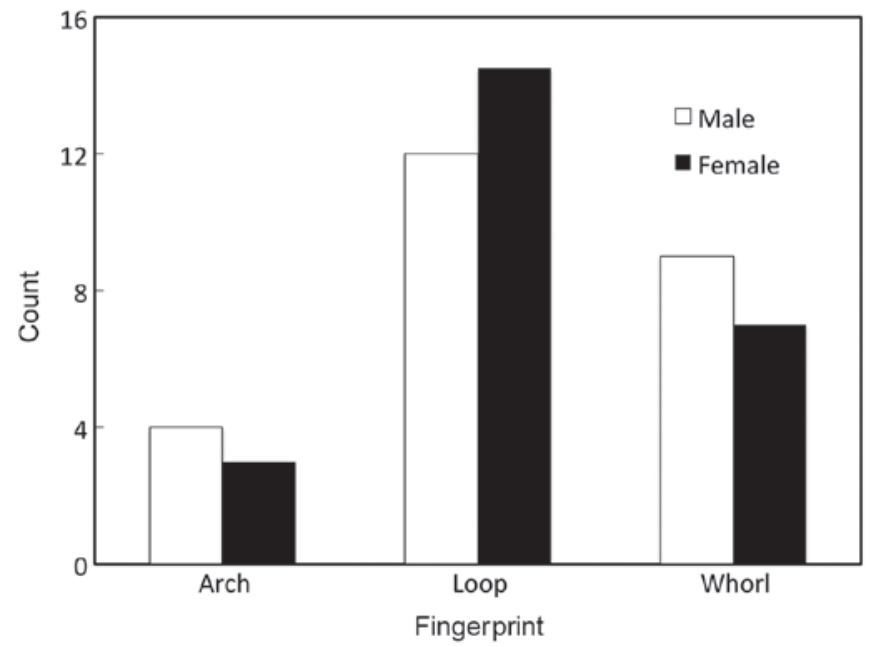

Figure 5. Distribution of fingerprint patterns in males and females.

which the value was considered to indicate a female. A total of 21 males were accurately identified as males and 6 females were correctly identified as females (Table I). P-values revealed that there was no statistically significant variation in mandibular canine index between males and females $(\mathrm{P}=0.345)$.

\section{Discussion}

Gender determination is an important step in medico-legal examination and personal identification $(15,16)$. Personal identification is an essential component in the detection of unknown deceased people in suicide, homicide, accidents and mass disasters. It is also important in the case of a missing person or an individual hiding their identity (1). Various methods are used in gender identification, including cheiloscopy (study of lip prints) (1), dermatoglyphics (study of fingerprints) (1), and odontometrics (the measurement and study of tooth size) (17).

Cheiloscopy is a forensic tool that deals with the identification of humans based on lip traces (4). Lip prints are unique and do not change during the life of a person (18). Following the analysis of the predominant patterns of lip prints in males and females, the results demonstrated that Type I and Type I' lip patterns were predominant in females, and Type IV lip patterns were predominant in males. A similar predominance of lip prints was seen in a study conducted by Vahanwala et al (19), which showed that Types I and I' were the predominant types in females, and Types III and IV were predominant in males. Conversely, a study conducted by Sandhu et al (20) revealed that Type I lip patterns were predominant in both males and females.

Fingerprinting is the most widely used and best method for personal identification (21), since fingerprints are unique to an individual and do not change over the course of their lifetime, even after superficial injury to the fingers. Analysis of the predominant patterns of fingerprints in males and females in the present study revealed that the finger loop pattern of prints were predominant both in males and females. A similar predominance was observed in a study conducted by Nagasupriya et al (1), who also reported a predominance of loop patterns in males and females. A study by 
Rastogi and Pillai (22) reported that the whorl pattern was predominant in males, and the loop pattern in females.

Canines are considered to exhibit the greatest sexual dimorphism among all types of teeth (23), and are the least affected by dental caries (11). The present study evaluated the efficacy of the mandibular canine index in gender determination. The results demonstrated that the mandibular canine index is not significant in gender determination, results which were concordant with those reported by Acharya and Mainali (24). Conversely, studies by Duraiswamy et al (25) and Wadhwan et al (12) reported that the mandibular canine index is a significant and reliable tool for the determination of gender.

The results of the present study demonstrated that Types I and I' are the predominant types of lip patterns in females, and Type IV is the predominant pattern in males. The loop fingerprint pattern was predominant in males and females. Mandibular canine index was not found to be a significant indicator of gender. To conclude, lip prints were determined to be unique, and exhibited sexual dimorphism based on their patterns in males and females, whereas fingerprint patterns were similar in males and females. Therefore, lip prints may be a potential forensic tool for gender determination and are considered superior to other tools, including finger prints and mandibular canine index.

\section{References}

1. Nagasupriya A, Dhanapal R, Reena K, Saraswathi TR and Ramachandran CR: Patterns-'A crime solver'. J Forensic Dent Sci 3: 3-7, 2011.

2. Saxena S, Sharma P and Gupta N: Experimental studies of forensic odontology to aid in the identification process. J Forensic Dent Sci 2: 69-76, 2010

3. Sivapathasundharam B, Prakash PA and Sivakumar G: Lip prints (cheiloscopy). Indian J Dent Res 12: 234-237, 2001.

4. Sharma P, Saxena S and Rathod V: Cheiloscopy: The study of lip prints in sex identification. J Forensic Dent Sci 1: 24-27, 2009.

5. Kasprzak J: Possibilities of cheiloscopy. Forensic Sci Int 46: $145-151,1990$

6. Venkatesh R and David MP: Cheiloscopy: An aid for personal identification. J Forensic Dent Sci 3: 67-70, 2011.
7. Vats Y, Dhall JK and Kapoor AK: Gender variation in morphological patterns of lip prints among some north Indian populations. J Forensic Dent Sci 4: 19-23, 2012.

8. Gyula G: A short history and some results of the dermatoglyphic studies in Hungary. Acta Biol Szeged 44:135-138, 2000.

9. Kücken $M$ and Newell AC: Finger print formation. J Theor Biol 235: 71-83, 2005.

10. Vij K (ed): Textbook of Forensic Medicine and Toxicology. 3rd edition. Elsevier, New Delhi, 89-91, 2005.

11. Kaushal S, Patnaik VVG and Agnihotri G: Mandibular canines in sex determination. J Anat Soc India 52: 119-124, 2003.

12. Wadhwan V, Aadithya B Urs and Manchanda A: Sex determination using three methodologies as a tool in forensic dentistry. JIDA 5: 77-80, 2011

13. Boaz K and Gupta C: Dimorphism in human maxillary and mandibular canines in establishment of gender. J Forensic Dent Sci 1: 42-44, 2009.

14. Tsuchihashi Y: Studies on personal identification by means of lip prints. Forensic Sci 3: 233-248, 1974.

15. Srivastava PC: Correlation of odontometric measures in sex determination. J Indian Acad Forensic Med 32: 56-61, 2010.

16. Singh J, Gupta KD, Sardana V, Balappanavar AY and Malhotra G: Sex determination using cheiloscopy and mandibular canine index as a tool in forensic dentistry. J Forensic Dent Sci 4: 70-74, 2012.

17. Iscan MY and Kedici PS: Sexual variation in bucco-lingual dimensions in Turkish dentition. Forensic Sci Int 137: 160-164, 2003.

18. Gondivkar MS, Indurkar A, Degwekar S and Bhowate R: Cheiloscopy for sex determination. J Forensic Dent Sci 1: 56-59, 2009.

19. Vahanwala S, Nayak CD and Pagare SS: Study of lip prints as aid for sex determination. Medico-Legal Update 5: 93-98, 2005.

20. Sandhu SV, Bansal H, Monga P and Bhandari R: Study of lip print pattern in a Punjabi population. J Forensic Dent Sci 4: 24-28, 2012.

21. Kaur R, Mazumdar SG and Bhonsle D: A study on various methods of gender identification based on fingerprints. Int J Emerg Technol Adv Engineering 2: 532-537, 2012.

22. Rastogi $P$ and Pillai KR: A study of fingerprints in relation to gender and blood group. J Indian Acad Forensic Med 32: 11-14, 2010.

23. Acharya $A B$ and Mainali S: Univariate sex dimorphism in the Nepalese dentition and the use of discriminant functions in gender assessment. Forensic Sci. Int 173: 47-56, 2007.

24. Acharya AB and Mainali S: Limitations of mandibular canine index in sex assessment. J Forensic Leg Med 16: 67-69, 2009.

25. Duraiswamy P, Tibdewal H, Patel K, Kumar S, Dhanni C and Kulkarni S: Sex determination using mandibular canine index in optimal-fluoride and high-fluoride areas. J Forensic Dental Sci 1: 99-103, 2009. 